Albitzky: Ưber Oxydation ungesättigter Säuren etc. 357

\title{
Untersuchungen ans dem chemischen Laboratorium von Prof. Alexander Saytzeff zu Kasan.
}

\section{0. Über die Oxydation nngesättigter Sänren mit Caros Reagens; \\ von}

\section{A. Albitzky.}

Die in der vorhergehenden Abhandlung beschriebenen Versuche führten zu dem Schlusse, daß bei der Oxydation (Hydratation) ungesättigter stereoisomerer Säuren Dioxysäuren erhalten werden können, die einer anderen stereoisomeren Reihe angehören, als die, welche durch Oxydation mit Kaliumpermanganat in alkalischer lösung resultieren, vorausgesetzt, $\mathrm{da} B$ die Oxydation in saurer Lösung vorgenommen wird. Weitere Versuche bestätigten die Voraussetzung.

Es war schwierig, ein saures Oxydationsmittel ausfindig zu machen, das nicht sehr energisch wirken sollte, da sonst eine Zerstörung der Moleküle stattfinden würde.

$\mathrm{Zu}$ den ersten Oxydationsversuchen, die man mit Őlsäure vornahm, wurde Jodsäure verwendet. Mit ihr führte Brezinsky ${ }^{1}$ ) nach dem Vorschlage von $L$ w off die Oxydation von Amylen (aus tertiärem Amyljodid) und von Isobutylen aus. Er erhielt aus Amylen, auber tertiärem Amylalkohol, Amylenglykol, aus Isobutylen aber Isobutylenglykol und Trimethylkarbinol. Diese Versuche waren jedoch nicht besonders erfolgreich, weshalb ein neues Oxydationsmittel, das sogenannte Carosche Reagens angewendet wurde.

Die Reaktionen, die mit diesem Reagens angestellt werden, sind äuBerst mannigfaltig; unter anderem kann es auf Verbindungen einwirken, die eine zweifache Bindung enthalten, ähnlich einer schwachen Lösung von Kaliumpermanganat. So erhielt Baeyer2), indem er mit ihm Terpineol oxydierte,

1) Journ. d. russ. phys.-chem. Gres. 21, 354.

2) Ber. 32, 3633. 
358 Albitzky: Ũber Oxydation ungesättigter Säuren otc.

Trioxyhexahydrocymol, dasselbe, das Wallach bei Oxydation von Terpineol durch Kaliumpermanganat erzielt hatte.

Damit die Reaktion leichter zu verfolgen sei, wurde für die Anfangsversuche ein Material verwendet, das in reiner Qualität am leichtesten zu erlangen ist, und zwar die Elaidinsäure. Außer diesem Umstande fiel die Wahl auf diese Säure noch aus dem Grunde, daB eine Dioxystearinsäure rom Schmelzp. 131,5 ${ }^{\circ}$, deren Entstehung hierbei zu erwarten war, am leichtesten unter den Reaktionsprodukten nachzuweisen ist. Nach einigen mißlungenen Versuchen wurde nachfolgender Weg zum Zweck der Oxydation eingeschlagen.

\section{Oxydation der Elaidinsäure.}

Eine Säure vom Erstarrungsp. $45^{\circ}$ wurde verwendet. Die Quantität der Säure wurde ebenso wie die des Oxydationsmittels nach den Angaben von Baeyer und Villiger für die Oxydation von Terpineol berechnet, d. h. es wurden $18 \mathrm{~g}$ Elaidinsäure, $22 \mathrm{~g}\left(\mathrm{NH}_{4}\right)_{2} \mathrm{~S}_{2} \mathrm{O}_{8}$ und $20 \mathrm{~g} \mathrm{H}_{2} \mathrm{SO}_{4}$ verbraucht. Die Reaktion wurde in derselben Weise ausgeführt, wie sie bei der Einwirkung von Schwefelsäure auf Ölsäure zur Gewinnung von Oxystearinsäure angewendet wird. Die Elaidinsäure wurde in einer Schale geschmolzen und, als sie zu erstarren begann, mit kleinen Mengen eines Gemisches von feinzerriebenem Ammoniumpersulfat mit Schwefelsäure unter beständigem Umrühren mit dem Thermometer vermengt. Bei Zusatz des Oxydationsmittels hob sich die Temperatur des Gemisches, die an den Rändern erstarrte Säure schmolz, aus welchem Grunde es mitunter notwendig wurde, die Schale in Eiswasser zu stellen. Weitere Mengen des Oxydationsmittels wurden nur nach dem Sinken der Temperatur bis zur Anfangstemperatur zugesetzt. Nach Zusatz einer gewissen Menge des Oxydationsmittels erstarrte die Säure nicht mehr, und das Gemenge erhielt eine derart zähe Konsistenz, daß es schwer wurde, sie durchzurühren. Die Temperatur schwankte im Verlaufe der Reaktion von $40^{\circ}-55^{\circ}$. Das Produkt der Reaktion, von hellbrauner Färbung, bis zum nächsten Tage an einem kalten Orte belassen, wurde nachher in Wasser ausgegossen, in dem es sich bei Umrühren vollständig löste; es bildete sich nur eine geringe weiße Trübung. Beim Kochen 
Albitzky: Ưber Oxydation ungesättigter Säuren etc. 359

dieser wäßrigen Lösung zeigte sich allmählich die Bildung von Öl, das zu einer teigigen Masse erstarrte. In Anbetracht der Möglichkeit einer Bildung von anhydridischen Substanzen beim Kochen mit Schwefelsäure wurde das Produkt der Reaktion mit starker Ätzkalilösung verseift, wobei es beständig umgerührt und auf dem Sandbade bis zur Trockne erhitzt wurde. Nachher wurde das ganze in Wasser gelöst und durch Salzsäure zerlegt. Die ausgeschiedenen Säuren, abfiltriert, ausgedrückt und aus Alkohol umkristallisiert, ergaben eine erste Fraktion von $5 \mathrm{~g}$ Gewicht, die nach Auswaschen auf dem Filter mit Äther einen Schmelzpunkt von $127^{\circ}-129^{\circ}$, Erstarrungsp. $119^{\circ}-114^{\circ}$ aufwies; aus der Mutterlauge konnten weiter keine Kristalle eliminiert werden. Nach dem Umkristallisieren aus Äther schmolz das gewonnene Produkt bei $129^{\circ}-132^{\circ}$ und erstarrte bei $125^{\circ}-123^{\circ}$.

1. $0,137 \mathrm{~g}$ Substanz gaben $0,34 \mathrm{~g} \mathrm{CO}_{2}$ und $0,141 \mathrm{~g} \mathrm{H}_{2} \mathrm{O}$.

2. 0,134 g Substanz gaben $0,322 \mathrm{~g} \mathrm{CO}_{2}$ und $0,1395 \mathrm{~g} \mathrm{H}_{2} \mathrm{O}$.

Berechnet für $\mathrm{C}_{18} \mathrm{H}_{38} \mathrm{O}_{4}$ :

Gefunden:

$\begin{array}{cccc}\mathrm{C} & & 1 . & 2 . \\ \mathrm{H} & 68,27 & 67,68 & 67,58 \% \\ \mathrm{~A} & 11,49 & 11,53 & 11,68 \% .\end{array}$

Aus der Analyse ist zu ersehen, dab die untersuchte Substanz nichts weiter als Dioxystearinsäure vorstellt. Der etwas zu geringe Gehalt an Kohlenstoff läBt sich durch Beimischung irgend welcher Substanzen erklären, die möglicherweise Schwefelsäure enthalten. Infolge dessen wurde die Substanz abermals

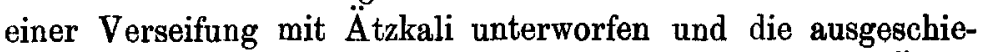
dene Säure durch Umkristallisieren aus Alkohol und Äther gereinigt. $\mathrm{Zu}$ einer neuen Analyse wurde eine Fraktion vom Schmelzp. $130^{\circ}-132^{\circ}$, Erstarrungsp. $124^{\circ}-121^{\circ}$, verwendet; dieselbe ergab:

0,1335 $\mathrm{g}$ Substanz gaben 0,334 $\mathrm{g} \mathrm{CO}_{2}$ und $0,141 \mathrm{~g} \mathrm{H}_{2} \mathrm{O}$.

$\begin{array}{ccc} & \text { Berechnet für } \mathrm{C}_{18} \mathrm{H}_{38} \mathrm{O}_{4}: & \text { Gefunden: } \\ \mathrm{C} & 68,27 & 68,23 \% \\ \mathrm{H} & 11,49 & 11,83 \% .\end{array}$

Die gewonnene Substanz ist Dioxystearinsäure; nach allen ihren äuBeren Merkmalen stimmt sie genau mit der Dioxystearinsäure, die durch Oxydation der Ölsäure resultiert; überein, nur ist ihre Schmelzpunkttemperatur etwas niedriger. 
Es muß hierbei aber in Betracht gezogen werden, dab die durch Oxydation der Ölsäure resultierende Dioxystearinsäure unter gewöhnlichen Umständen bei $132^{\circ}-134^{\circ}$ schmilzt, um aber einen Schmelzp. $136,5^{\circ}$ zu erzielen, muß sie einem gründlichen Reinigungsproze $B$ unterworfen werden.

Um allen $\mathrm{Zweifeln}$ der Identität dieser Dioxystearinsäure mit der schon bekannten zu begegnen, wurde eine Bestimmung ihrer Löslichkeit in Alkohol vorgenommen. Zu diesem Zwecke wurde die analysierte Säure in warmem Alkohol von $99,5^{\circ}$ (nach Tralles) gelöst, die Lösung durchfiltriert und bis zum anderen Tage in einem verkorkten Kolben stehen gelassen. Bevor man am anderen Tage die Lösung für die Löslichkeitsbestimmung von den auskristallisierten Teilen der Substanz abfiltrierte, wurde der Kolben in der Zeit von wenigstens $1 \frac{1}{2}$ Stunden mehrmals durchgeschüttelt; die Temperatur veränderte sich während dieser Zeit nicht.

Die Löslichkeitsbestimmung ergab:

9,42 $\mathrm{g}$ Lösung in Alkohol 99,5 $5^{\circ} \mathrm{Tr}$. bei $18^{\circ}$ hinterließen nach Verdunstung des Alkohols und Trocknen im Exsikkator bis zum beständigen Gewicht $0,06 \mathrm{~g}$; folglich lösen 100 Teile Alkohol bei $18^{\circ} 0,63 \mathrm{~g}$ Teile Dioxystearinsäure. Dieser Wert $0,63 \mathrm{~g}$ steht sehr nahe $0,59 \mathrm{~g}$, der Ziffer des Löslichkeitsverhältnisses in dem gleichen Alkohol bei $19^{\circ}$, die von Spiridon off ${ }^{1}$ ) für Dioxystearinsäure aus Ölsäure und Kaliumpermanganat gefunden worden ist.

Bei weiteren Versuchen ergab sich, daß eine Verseifung mit Ätzkali nicht absolut notwendig ist, da Wasser an und für sich bei gewöhnlicher oder Kochtemperatur auf jenes in Wasser lösliche Produkt der Einwirkung von Caros Reagens auf Elaidinsäure wirkt, wobei Dioxystearinsäure gebildet wird. Die Verseifung mit Ätzkali bewirkt nur eine Vergrößexung der Ausbeute an Dioxysäuren.

Weiter wurde folgender Versuch angestellt: Die Quantität der zur Reaktion verwendeten Substanzen, sowie die Bedingungen, unter denen sie ausgeführt wurde, waren dieselben, wie beim ersten Versuche, ein Unterschied trat nur darin ein, daB die Zerlegung durch Wasser nicht am anderen Tage,

1) Dies. Journ. [2] 40, 244. 
Albitzky: Über Oxydation ungesättigter Säuren ete. 361

sondern nach 2 Stunden ausgeführt wurde. Anfangs hatte man Eiswasser zuzusetzen, um die Schwefelsäure und ihre Salze zu entziehen; das Produkt der Reaktion schied sich als halbflüssige, in Wasser fast unlösliche Masse aus, als jedoch die ersten Waschwässer, die Schwefelsäure und deren Salze enthielten, abfiltriert waren, löste sich das Produkt nach verhältnismäBig nicht lang dauernder Mischung mit Wasser ziemlich leicht gänzlich in letzterem; es verblieb nur eine geringe Menge weißer Substanz, von der die Lösung schließlich abfiltriert wurde.

Der Rückstand auf dem Filter erwies sich als Dioxystearinsäure; nach Umkristallisieren aus Alkohol und Abwaschen mit Äther schmolz sie bei $128^{\circ}-130^{\circ}$, erstarrte bei $124^{\circ}-121^{\circ}$.

Die Lösung wurde durchgekocht, wobei sich ein Öl ausschied, das beim Erkalten erstarrte. Aus demselben resultierte beim Umkristallisieren aus Alkohol, später aus Äther, der mit wenig Alkohol versetzt war, wiederum Dioxystearinsäure vom Schmelzp. $127^{\circ}-130^{\circ}$, Erstarrungsp. $123^{\circ}-119^{\circ}$.

$\mathrm{Um}$ die Ausbeute der Dioxystearinsäure zu bestimmen, wurde noch ein Versuch zwecks Oxydation derselben Quantität $(18 \mathrm{~g})$ Elaidinsäure angestellt, bei dem alle Operationen möglichst peinlich ausgeführt wurden. Die Lösung des Zwischenproduktes, das nach Auswaschen der Schwefelsäure erhalten war, wurde einer andauernden Kochprozedur bis zur vollen Zerlegung unterworfen.

Das ausgeschiedene $\mathrm{Ol}$ erstarrte zu einer wachsartigen Masse, es wurde mit KOH verseift. Nach dem Umkristallisieren der ausgeschiedenen Säuren aus Alkohol resultierten $8 \mathrm{~g}$ einer Substanz, die, nach Auswaschen auf dem Filter mit Äther den Schmelzp. bei $127^{\circ}-129^{\circ}$, Erstarrungspunkt bei $120^{\circ}-118^{\circ}$ zeigte.

Bei weiteren Versuchen der Oxydation wie der Elaidinsäure, so auch anderer Säuren, wurde das Produkt der Einwirkung von Caros Reagens meist nicht am anderen Tage, sondern nach 2 Stunden zerlegt. Fernerhin, wenn auf diesem Wege irgend eine Dioxysäure dargestellt worden war, wurde das Produkt der Reaktion, nach Auswaschen der Schwefel- 
362 Albitzky: Ǔber Oxydation ungesättigter Säuren etc. säure, nicht in Wasser gelöst, sondern direkt mit Ätzkali verseift.

$$
\text { Oxydation der Ölsäure. }
$$

$\mathrm{Zu}$ diesen Versuchen wurde eine Săure verwendet, die aus Mandelöl gewonnen und von den festen Säuren durch die Löslichkeit der Bleisalze in Äther getrennt war. Die Quantität der verwendeten Substanzen war: $18 \mathrm{~g}$ Ölsäure, $22 \mathrm{~g} \mathrm{NH}_{4} \mathrm{~S}_{2} \mathrm{O}_{8}$ und $20 \mathrm{~g} \mathrm{H}_{2} \mathrm{SO}_{4}$.

Die Ölsäure wurde bis zum Beginn der Kristallbildung, ebenso auch das Carosche Reagens, durch Eiswasser abgekühlt. Der Oxydationsprozeß ging unter denselben Bedingungen vor sich, wie bei der Elaidinsäure; die Temperatur schwankte während der Reaktion zwischen $10^{\circ}-25^{\circ}$; die Zerlegung durch Eiswasser wurde eine Stunde nach der Oxydation ausgeführt. Das ausgeschiedene Öl wurde von dem Schwefelsäure und Salze enthaltenden Wasser getrennt und nachher mit neuer Wassermenge behandelt, in dem sich alles, außer einem geringen weißen Bodensatz, löste. Aus der dunkelbraun grünen eingedampften Lösung schied sich ein dunkelbraunes Öl aus, das beim Erkalten erstarrte. Es besaß eine Konsistenz, ähnlich der Kuhbutter. Nach Unkristallisieren aus Alkohol gab es nur eine Fraktion von gelblicher Farbe, daher wurde diese nochmals aus Alkohol umkristallisiert, wobei $2 \mathrm{~g}$ resultierten. Nachdem die Substanz aus Äther umkristallisiert war, zeigte sie den Schmelzp. $95^{\circ}-98^{\circ}$, Erstarrungsp. $85^{\circ}$ bis $83^{\circ}$; zur Analyse wurde aus ihr dureh Omkristallisieren aus Äther $1 \mathrm{~g}$ einer Fraktion vom Schmelzp. 95 ${ }^{\circ}-98,5^{\circ}$, Erstarrungsp. $86^{\circ} \ldots 84^{\circ}$ gewonnen.

$0,121 \mathrm{~g}$ Substanz gaben $0,3035 \mathrm{~g} \mathrm{CO}_{2}$ und $0,126 \mathrm{~g} \mathrm{H}_{2} \mathrm{O}$.

$\begin{array}{ccc} & \text { Berechnet für } \mathrm{C}_{16} \mathrm{H}_{36} \mathrm{O}_{4}: & \text { Gefunden: } \\ \mathrm{C} & 68,27 & 68,4 \% \\ \mathrm{H} & 11,49 & 11,67 \% .\end{array}$

Die Resultate der Analyse zeigen, dab die gewonnene Substanz, ihrer Zusammensetzung nach, eine Dioxystearinsäure ist, und zwar, der Schmelzpunktstemperatur nach zu urteilen, eben jene, die durch Oxydation von Elaidinsäure mit Kaliumpermanganat erhalten wird. 
Albitzky: Über 0xydation ungesättigter Säuren etc. 363

Zum Beweise der Identität dieser, auf neuem Wege dargestellten Dioxystearinsäure, wurde eine Bestimmung ihrer Löslichkeit in Alkohol vorgenommen. Zu gleicher Zeit wurde auch die Löslichkeitsbestimmung der Dioxystearinsäure ausgeführt, die durch Oxydation der Elaidinsäure mit Kaliumpermanganat resultierte, da eine solche bis dahin noch nicht bestimmt worden war. Diese Säure hatte den Schmelzp. $96^{\circ}$ bis $98^{\circ}$, Erstarrungsp. $87^{\circ}-86^{\circ}$.

1. $3,357 \mathrm{~g}$ Lösung einer Dioxystearinsäure vom Schmelzp. $96^{\circ}-99^{\circ}$, Erstarrungsp. $87^{\circ}-86^{\circ}$, aus Elaidinsäure in Alkohol 99,5 Tr. bei $18^{\circ}$ gesättigt, gaben $0,118 \mathrm{~g}$ im Exsikkator bis zum unveränderlichen Gewichte getrockneten Rückstand. Hieraus wurde berechnet, daß 100 Teile Alkohol 3,64 Teile der Säure zu lösen fähig sind.

z. $4,435 \mathrm{~g}$ Lösung einer Dioxystearinsäure vom Schmelzp. $9^{\circ}$ bis $98,5^{\circ}$, Erstarrungsp. $86^{\circ}-85^{\circ}$, aus Ölsäure unter denselben Bedingungen gaben $0,188 \mathrm{~g}$ Rückstand; folglich konnten in 100 Teilen 4,42 Teile gelöst werden.

Der Unterschied zwischen den Resultaten beider Bestimmungen ist ziemlich merklich, aber doch müssen, nach allem zu urteilen, wie auch nach der Analogie des Verhaltens der Dioxybehensäuren, beide Dioxystearinsäuren als identisch betrachtet werden. Die etwas größere Löslichkeit der Dioxystearinsäure aus Ölsäure muß ihrer geringen Reinheit zugeschrieben werden; die Ausbeute an kristallinischen Sub. stanzen ist in diesem Falle sehr gering, und es fällt sehr schwer, sie rein zu erhalten.

Die geringe Ausbeute an Dioxystearinsäure beim eben beschriebenen Versuche der Oxydation von Ölsäure, konnte durch die schädliche Einwirkung des andauernden Kochens bei Gegenwart nicht volllkommen ausgewaschener Schwefelsäure erklärt werden.

Daher wurde noch ein Versuch mit derselben Quantität von Ölsäure, unter genau denselben Bedingungen angestellt, nur mit dem Unterschiede, daß das nach Zerlegung durch Wasser ansgeschiedene Öl nicht in Wasser gelöst, sondern direkt mit KOH verseift wurde. Nach mehrmaligem Umkristallisieren, anfangs aus Alkohol, später aus Äther, gaben die ausgeschiedenen Säuren gegen 1,5 g Dioxystearinsäure vom Schmelzp. $95^{\circ}-97^{\circ}$, Erstarrungsp. $86^{\circ}-85^{\circ}$. Wie zu er- 
364 Albitzky: Über Oxydation ungesättigter Säuren etc.

sehen, vergrößerte sich die Ausbeute an Dioxysäure hierbei, aber doch blieb sie verhältnismäßig unbedeutend.

\section{Oxydation der Erucasäure.}

Die Menge der zur Reaktion verwendeten Erucasäure vom Erstarrungsp. $34^{\circ}$, ebenso wie die des Oxydationsmittels, war dieselbe, wie die der Elaidinsäure.

Es wurden $22 \mathrm{~g} \mathrm{C}_{22} \mathrm{H}_{42} \mathrm{O}_{2}, 22 \mathrm{~g}\left(\mathrm{NH}_{4}\right)_{2} \mathrm{~S}_{2} \mathrm{O}_{8}$ und $20 \mathrm{~g}$ $\mathrm{H}_{2} \mathrm{SO}_{4}$ verwendet. Die Reaktion der Oxydation wurde ebenso ausgeführt, wie oben beschrieben, d. h. zur Erucasäure, beim Beginn der Kristallbildung, wurde in kleinen Mengen das Carosche Reagens unter beständigem Umrühren zugesetzt. Die Temperatur schwankte während der Reaktion zwischen $34^{\circ}$ bis $42^{\circ}$. Die Zerlegung durch Wasser wurde am nächsten Tage ausgeführt, die ersten Waschwässer abfiltriert, das ausgeschiedene öl aber direkt mit Wasser durchgekocht, ohne seine Lösung zu erwarten. Das beim Abkühlen erstarrte Produkt wurde dann mit KOH verseift. Nach zweimaligem Umkristallisieren der ausgeschiedenen Säuren resultierten $11 \mathrm{~g}$ Substanz vom Schmelzp. $95^{\circ}-100^{\circ}$, Erstarrungsp. $93^{\circ}-84^{\circ}$; aus dieser aber für die Elementaranalyse durch zweimaliges Umkristallisiren aus Alkohol, wobei die Rristalle auf dem Filter mehrmals mit Äther ausgewaschen wurden, $6 \mathrm{~g}$ einer Fraktion vom Schmelzp. $98^{\circ}-100^{\circ}$, Erstarrungsp. $92^{\circ}-91^{\circ}$. 0,1275 g Substanz gaben $0,332 \mathrm{~g} \mathrm{CO}_{2}$ und $0,14 \mathrm{~g} \mathrm{H}_{2} \mathrm{O}$.

$\begin{array}{ccc} & \text { Berechnet für } \mathrm{C}_{22} \mathrm{H}_{44} \mathrm{O}_{4}: & \text { Gefunden: } \\ \mathrm{C} & \mathbf{7 0 , 8 8} & \mathbf{7 1 , 0 2 \%} \% \\ \mathrm{H} & 11,93 & \mathbf{1 2 , 3 1} \% \text {. }\end{array}$

Die Resultate der Analyse zeigen, daß die gewonnene Substanz Dioxybehensäure ist, und zwar nach der Schmelztemperatur, die aus Brassidinsäure durch Oxydation mit Kaliumpermanganat entstehende.

Zum Beweise ihrer Identität wurde eine Bestimmung der Löslichkeit der auf beiden Wegen gewonnenen Dioxybehen. säuren vorgenommen. Für diese Bestimmung wurde eine Probe der Säure aus der Sammlung des Laboratoriums verwendet.

1. $8,61 \mathrm{~g}$ bei $17^{\circ}$ gesättigter alkoholischer, 99,5 Tr., Lösung einer Dioxybehensäure vom Schmelzp. $99^{\circ}-101^{\circ}$, Erstarrungsp. $92^{\circ}-89^{\circ}$ aus 
Albitzky: Ũber 0xydation ungesättigter Säuren etc. 365

Brassidinsäure hinterließen nach Verdunsten von Alkohol und Austrocknen des Rückstandes im Exsikkator bis zum beständigen Gewicht $0,0745 \mathrm{~g}$. Folglich lösen sich in 100 Teilen Alkohol 0,87 Teile Säure.

2. 10,151 g ebensolcher, bei derselben Temperatur gesättigter Lösung einer Dioxybehensäure vom Schmelzp. $98^{\circ}-100^{\circ}$, Erstarrungsp. $92^{\circ}$ bis $91^{\circ}$ aus Erukasäure ließen $0,09 \mathrm{~g}$ trockenen Rückstandes zurück. Folglich lösen sich bei $17^{\circ}$ in 100 Teilen Alkohol 0,89 Teile Säure.

Die bei den Löslichkeitsbestimmungen erhaltenen GröBen stehen einander so nahe, daß sie genügend die Identität der auf beiden Wogen gewonnenen Dioxybehensäure beweisen.

\section{Oxydation der Brassidinsäure.}

Der Versuch der Oxydation der Brassidinsäure vom Erstarrungsp. $60^{\circ}$ wurde mit denselben Quantitäten und unter denselben Bedingungen, wie die Oxydation der Erukasäure ausgeführt, d. h. es wurden $22 \mathrm{~g} \mathrm{C}_{22} \mathrm{H}_{42} \mathrm{O}_{2}, 22 \mathrm{~g}\left(\mathrm{NH}_{4}\right)_{2} \mathrm{~S}_{2} \mathrm{O}_{8}$ und $20 \mathrm{~g}_{2} \mathrm{SO}_{4}$ verwendet.

Der Anfang der Reaktion verlief bei $63^{\circ}$; da hierbei der größte Teil der Brassidinsäure an den Rändern der Schale erstarrte, so muBte sie mit dem Spatel abgelöst werden, bald jedoch hörte das Erstarren auf; die Temperatur schwankte $z$ wischen $57^{\circ}$ und $70^{\circ}$. Am nächsten Tage wurde das Reaktionsprodukt durch Wasser zerlegt und dann abfiltriert. Da das Lösen des erhaltenen Produktes in dem neu hinzugegossenen Wasser sehr langsam vor sich ging, so wurde alles einfach durchgekocht, daraufhin mit $\mathrm{KOH}$ verseift und durch $\mathrm{HCl}$ zerlegt. Die ausgeschiedene Säure wog nach zweimaligem Umkristallisieren $12 \mathrm{~g}$ und schmolz bei $126^{\circ}-127,5^{\circ}$, erstarrte bei $119^{\circ}-114^{\circ}$. Hieraus wurde für die Analyse durch zweimaliges Umkristallisieren aus Alkohol nach mehrmaligem Auswaschen der Kristalle auf dem Filter mit Äther $7 \mathrm{~g}$ einer Fraktion vom Schmelzp. $131^{\circ}-133^{\circ}$, Erstarrungsp. $123^{\circ}-121^{\circ}$ ausgeschieden.

0,1295 g Substanz gaben $0,335 \mathrm{~g} \mathrm{CO}_{2}$ und $0,1395 \mathrm{~g} \mathrm{H}_{2} \mathrm{O}$.

$\begin{array}{ccc} & \text { Berechnet für } \mathrm{C}_{22} \mathrm{H}_{44} \mathrm{O}_{4}: & \text { Gefunden: } \\ \mathrm{C} & \mathbf{7 0 , 8 8} & \mathbf{7 0 , 5 5 \%} \% \\ \mathrm{H} & \mathbf{1 1 , 9 3} & \mathbf{1 2 , 0 7 \%} \%\end{array}$

Aus den Resultaten der Analyse und der Schmelztemperatur folgt, daB die Substanz dieselbe Dioxybehensäure 
366 Albitzky: Über 0xydation ungesättigter Säuren etc.

ist, die man durch Oxydation der Erukasäure mit Kaliumpermanganat erhält.

Es wurden Bestimmungen der Löslichkeit in Alkohol sowohl dieser, wie auch der aus Erukasäure erhaltenen Dioxybehensäure vorgenommen.

1. $17,7825 \mathrm{~g}$ einer bei $18^{\circ}$ gesättigten, alkoholischen $\left(99,5^{\circ} \mathrm{Tr}\right.$.) Lösung einer Dioxybehensäure vom Schmelzp. $130^{\circ}-131^{\circ}$, Erstarrungsp. $121^{\circ}-120^{\circ}$ aus Erukasäure gaben nach Verdunsten des Alkohols und Trocknen im Exsikkator bis zum unveränderlichen Gewichte $0,0245 \mathrm{~g}$ trockenen Rückstand. Folglich lösen sich in 100 Tln. Alkohol 0,13 Tle. Säure.

2. $21,565 \mathrm{~g}$ einer ebensolchen Lösung von Dioxybehensäure vom Schmelzp. $131^{\circ}-133^{\circ}$ und Erstarrungsp. $123^{\circ}-121^{\circ}$ aus Brassidinsäure gaben unter denselben Bedingungen $0,024 \mathrm{~g}$ trockenen Rückstandes; also lösen sich in 100 Teilen Alkohol 0,11 Teile Säure.

Aus allem ist $z u$ ersehen, daß bei der Oxydation durch das Carosche Reagens in saurer Lösung Reaktionen eintreten, die umgekehrt denen sind, die bei der Oxydation mit Kaliumpermanganat in alkalischer Lösung erfolgen.

Es ist hier am Platze, auch die Resultate der Vergleichung der Ausbeute von Dioxysäuren, gewonnen auf dem einen oder dem anderen Wege, anzuführen. Bei der Oxydation von Ölsäure mit $\mathrm{KMnO}_{4}$ ist die Ausbeute an Dioxystearinsäure mit hoher Schmelztemperatur gegen $64 \%$ der Theorie ${ }^{1}$ ), während sie bei der Oxydation von Elaidinsäure durch das Carosche Reagens gegen $40 \%$ beträgt. In Bezug auf die Ausbeute der Dioxystearinsäure vom niedrigeren Schmelzpunkt existieren in der Literatur keine Angaben; bei der Oxydation von Ölsäure durch das Carosche Reagens erhält man etwa 7,5\% Ausbeute. Die Ausbeute an Dioxybehensäuren bei Anwendung des Caroschen Reagens ist jedoch eine bedeutend größere, als bei der Einwirkung von $\mathrm{KMnO}_{4}$. So erhielt Urwanz o ff ${ }^{9}$ ) von Dioxybehensäure (Schmelzp. $127^{\circ}$ ) aus Erukasäure $27 \%$ der Theorie, während aus Brassidinsäure gegen $50 \%$ in derselben Reinheit erzielt wurden. Soukowsky ${ }^{3}$ ) erhielt durch Oxydation der Brassidinsäure

1) Dies. Journ. [2] 33, 304.

2) Dies. Journ. [2] 39, 335. $\quad$ s) Das. [2] 60, 70. 
Albitzky: Über 0xydation ungesättigter Säuren etc. 367 etwa $20 \%$ Dioxybehensäure von niedrigem Schmelzpunkt; durch Oxydation der Erukasäure mit Caros Reagens erhält man gegen $46 \%$.

Nachdem die Fähigkeit des Caroschen Reagens, die Bildung von Dioxysäuren zu bewirken, festgestellt war, war es wichtig, diese Reaktion näher zu erforschen und das Zwischenprodukt, aus dem schließlich die Dioxysäure hervorgeht, kennen zu lernen. Aber die zu diesem $Z_{\text {weeke angestellten Versuche }}$ ergaben nicht bestimmte Resultate; denn die fragliche Substanz veränderte sich leicht, so daB sie nicht einheitlich erhalten werden konnte, um ihre Struktur sicher zu bestimmen.

Beinahe alle diese Versuche wurden mit der Elaidinsäure angestellt. Da als Bauptzweck bei den Versuchen die Bestimmung der Zusammensetzung des in Wasser löslichen Zwischenproduktes der Einwirkung des Caroschen Reagens auf Elaidinsäure angestrebt wurde, so nahm man die Oxydation auf gewöhnlichem Wege vor; das entstandene Produkt wurde anfangs mit Wasser ausgewaschen und dann in solchem gelöst. Die durchsichtige abfiltrierte Lösung wurde mit Äther ausgezogen. Da dieses $\mathbf{Z}$ wischenprodukt allmählich eine Veränderung mit Wasser erfährt, also sich ein wenig Dioxystearinsäure ausscheidet, so wurde die ätherische Lösung einmal durch geschmolzenes schwefelsaures Natron, ein anderes mal durch Chlorcalcium getrocknet. Es stellte sich jedoch heraus, daß dieser Weg zum Zwecke des Trocknens nicht eingeschlagen werden durfte, weil der Rückstand nach Abdampfen des Äthers in beiden Fällen mineralische Beimischung enthielt. Bei den folgenden Versuchen wurde daher das fragliche Produkt durch Äther extrahiert, der Rückstand durch Auflösen in wasserfreiem Äther, Filtrieren und Austrocknen im Exsikkator in luftrerdünntem Raume gereinigt.

\section{Versuch I.}

Nach der Oxydation der Elaidinsäure und Lösung des Produktes in Wasser hatte sich beim Stehen bis zum nächsten Tage ein weißer Niederschlag ausgeschieden; das Filtrat von diesem wurde mit Äther ausgezogen. Beim Umkristallisieren 
368 Albitzky: Über Oxydation ungesättigter Säuren etc.

des Niederschlages aus Alkohol resultierte eine Substanz, die nach Auswaschen mit Äther bei $126^{\circ}-130^{\circ}$ schmolz und bei $121^{\circ}-116^{\circ}$ erstarrte, folglich Dioxystearinsäure. Aus der Mutterlauge konnten nicht mehr Kristalle erzielt werden, auch erstarrte sie nach dem Kochen mit Wasser nicht mehr; nachdem sie aber mit schwacher KOH-Lösung durchgekocht worden war, gab sie Dioxystearinsäure, die nach dem Umkrystallisieren aus Alkohol bei $122^{\circ}-125^{\circ}$ schmolz. - Das Filtrat wurde mit $\ddot{A}$ ther extrahiert, nach Abdampfen desselben verblieb als Rückstand eine ölige Substanz mit geringer Einlage von Kristallen. Nach dreitägigem Trocknen im Exsikkator wurden die Kristalle, deren Quantität sich vergröBert batte, abfiltriert. Das Filtrat, ein durchsichtiges, dicktlüssiges, hellgelbes Öl, begann bei weiterem Aufbewahren im Exsikkator wiederum Kristalle abzusondern, von denen es durch Auflösen in wasserfreiem $\ddot{A}$ ther befreit wurde. Der Äther wurde nachher im Exsikkator verdunstet; das Öl ging beim Aufbewahren vollständig in kristallinischen Zustand über, nahm innen eine gelbe, an der Oberfläche aber eine fast dunkelbraune Färbung an. Zum endgültigen Austrocknen wurde die kristallinische Masse auf Tonplatten gestrichen und nachher mehrmals mit $\ddot{A}$ ther ausgewaschen, in welchem die Kristalle sich nicht lösten. Die erhaltene kristallinische Substanz schmolz bei $119^{\circ}-124^{\circ}$, wobei sie sich zersetzte. Nach halbstündigem Erhitzen im Luftbade bei $135^{\circ}-140^{\circ}$ schmolz sie, erstarrte nachber schon nicht mehr und wurde in Äther leicht löslich. Nach Verseifen mit Ätzkali gab diese, bis $135^{\circ}-140^{\circ}$ erhitzte Substanz eine Dioxystearinsäure, die nach einmaligem Umkristallisieren aus Alkohol bei $124^{\circ}-126^{\circ}$ schmolz und bei $119^{\circ}-115^{\circ}$ erstarrte.

Der Äther, der zum Auswaschen verwendet worden war, hinterließ noch eine geringe Quantität einer halbkristallinischen Substanz, die nach Verseifen mit KOH Dioxystearinsäure gab, die nach Umkristallisieren aus Alkohol bei $124^{\circ}-126^{\circ} \mathrm{schmolz}$ und bei $120^{\circ}-117^{\circ}$ erstarrte.

Wie aus obigem zu ersehen, kann aus den Resultaten dieses Versuches nur geschlossen werden, daß das fragliche

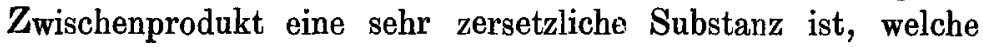
leicht eine mehr oder weniger reine Dioxystearinsäure giebt. Da also eine Garantie für die Einheitlichkeit der Substanz 
Albitzky: Uber 0xydation ungesättigter Säuren etc. 369 nicht vorhanden war, so war es auch nutzlos, eine Bestimmung von Kohlenstoff, Wasserstoff und Schwefel vorzunehmen.

\section{Versuch II.}

Noch ein Versuch zur Ausscheidung des fraglichen Produktes aus den Reaktionsprodukten wurde vorgenommen, jedoch ohne vorhergehende Auflösung desselben in Wasser. Nach Oxydation der Elaidinsäture wurde das Produkt nach 2 Stunden mit wasserfreiem $\ddot{A}$ ther behandelt.

In Äther lösten sich alle organischen Verbindungen mit Leichtigkeit, was daraus zu ersehen war, dab sich die auf dem Filter verbliebenen Salze vollkommen in Wasser lösten, wobei eine geringfügige Menge einer Substanz vom Schmelzp. $119^{\circ}$ bis $120^{\circ}$ zurückblieb. Beim Stehen der ätherischen Lösung bis zum nächsten Tage hatte sich ein ziemlich bedeutender weißer Bodensatz gebildet. Dieser wurde dann abfiltriert, zerrieben und mehrere Tage lang unter häufigem Umschütteln mit wasserfreiem Äther behandelt, nachher abfiltriert und mit Äther ausgewaschen. Eine Bestimmung der Schmelztemperatur zeigte, daß die Substanz bei $128^{\circ}-131^{\circ}$ schmolz, hierbei sich aber zersetzte.

1. $0,125 \mathrm{~g}$ Substanz gaben $0,3125 \mathrm{~g} \mathrm{CO}_{2}$ und $0,13 \mathrm{~g} \mathrm{H}_{2} \mathrm{O}$.

2. $0,134 \mathrm{~g}$ Substanz gaben $0,334 \mathrm{~g} \mathrm{CO}_{2}$ und $0,1405 \mathrm{~g} \mathrm{H}_{2} \mathrm{O}$.

Berechnet für $\mathrm{C}_{18} \mathrm{H}_{36} \mathrm{O}_{4}$ :

Gefunden:

$\begin{array}{cccc} & & 1 . & 2 . \\ \mathrm{C} & 68,27 & 68,18 & 67,95 \% \\ \mathrm{H} & 11,49 & 11,65 & 11,73 \% .\end{array}$

Um zu bestimmen, welche Veränderung die fragliche Substanz in ihrer Zusammensetzung beim Erhitzen erleidet, wurde eine geringe Quantität von ihr eine halbe Stunde lang im Luftbade bis $140^{\circ}$ erhitzt. Nachdem sie bei dieser Temperatur geschmolzen war, erstarrte sie nicht mehr und war in Äther leicht löslich. Die ätherische Lösung wurde abfiltriert; nach Verdunsten des Äthers und Trocknen im Exsikkator wurde die Substanz analysiert.

$0,16 \mathrm{~g}$ Substanz gaben $0,4225 \mathrm{~g} \mathrm{CO}_{8}$ und $0,164 \mathrm{~g} \mathrm{H}_{\mathrm{z}} \mathrm{O}$.

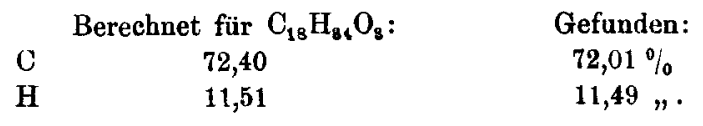

Journal f. prakt. Chemie [2] Bd, 67. 
370 Albitzky: Über 0xydation ungesättigter Säuren etc.

Aus den Analysen ist zu entnehmen, daß jene Substanz, die sich aus der ätherischen Lösung ausgeschieden hatte, bis zum Prozeß des Erhitzens nicht ganz reine Dioxystearinsäure vorstellt, nach diesem ProzeB aber irgend ein Anhydrid von ihr. Die Bildung dieses letzteren könnte dadurch erklärt werden, daß aus der ätherischen Lösung, außer Dioxystearinsäure, sich noch geringe Mengen des Zwischenproduktes ausscheiden, das als eine Verbindung der Elaidinsäure mit Caros Reagens zu betrachten ist, und mit Äther nicht ganz ausgewaschen werden kann. Beim Erhitzen jedoch erfolgt durch Anwesenheit dieses Zwischenproduktes der Austritt eines Wassermoleküls.

$\mathrm{DaB}$ dieses wirklich sich so verhält, kann daraus ersehen werden, daß der andere Teil der analysierten, aber noch nicht erhitzten Substanz, mit Wasser durchgekocht, eine sich wenig mehr ändernde Schmelztemperatur zeigte. Mit ein und derselben Probe wurden mehrere Schmelzpunkt- und Erstarrungspunktbestimmungen vorgenommen: 1. Schmelzp. $125^{\circ}-126^{\circ}$, Erstarrungsp. $115^{\circ}-110^{\circ} ; 2$. Schmelzp. $122^{\circ}-125^{\circ}$, Erstarrungspunkt $111^{\circ}-110^{\circ}$; 3. Schmelzp. $117^{\circ}-121^{\circ}$.

Es ließ sich im ganzen als Regel ansehen, daB, wenn eine Substanz erhalten wurde, bei der die Schmelztemperatur sich änderte, man sie nur durch Umkristallisieren aus Alkohol zu reinigen nötig batte, damit sie eine beständige Schmelztemperatur annahm.

Nach Abdampfen des Äthers aus dem Filtrat des oben angeführten Bodensatzes verblieb ein Ö1, das beim Aufbewahren im Exsikkator braun wurde. Beim Verseifen mit $\mathrm{KOH}$ ergab es Dioxystearinsäure. Mit diesem Öl wurde folgender Versuch angestellt: Ein Teil von ihm wurde sogleich nach dem $\mathbf{A b}$ dampfen des Äthers mit Wasser durchgeschättelt, wobei alles. sich auflöste. Aus der Lösung begann sich beim Stehen ein Niederschlag zu bilden. Bei Bestimmung der Schmelztemperatur ergab sich, daB er bei $111^{\circ}-114^{\circ}$ schmolz, dabei sich aber zerlegte. Beim Kochen der wäBrigen Lösung schied sich ein gelbliches Öl aus, das nach Verseifen mit $\mathrm{KOH}$ Dioxystearinsäure vom Schmelzp. $123^{\circ}-125^{\circ}$, Erstarrungsp. $120^{\circ}-117^{\circ}$ ergab. Der übrige Teil bräunte sich nach einiger Zeit recht stark und war in Wasser nicht mehr löslich. 


\section{Versuch III.}

Da vorausgesetzt werden konnte, daß die beobachtete starke Veränderung von der Wirkung mechanisch mitgerissener Schwefelsäure oder Caroscher Säure abhängt, so wurde zum Zwecke der Darstellung der fraglichen Substanz, die von chemisch mit ihr nicht verbundenen Säuren frei sein sollte, folgender Versuch unternommen. Es wurde auf gewöhnlichem Wege eine Lösung der $Z_{\text {wischensubstanz hergestellt, die nach- }}$ her bis zur alkalischen Reaktion durch Pottasche in der Voraussetzung neutralisiert wurde, daß in stark verdünnter wäßriger Lösung nur die mineralischen Säuren neutralisiert würden, die organischen aber, wenn auch nicht ganz, so doch zum größten Teil frei bleiben würden. Die neutralisierte Lösung wurde durch $\ddot{\text { ther }}$ extrahiert, wobei eine geringe Menge Substanz erhalten wurde, die an Vaselin erinnerte; sie änderte sich auch trotz langen Trocknens im Exsikkator nicht und blieb in Äther leicht löslich.

1. $0,151 \mathrm{~g}$ Substanz gaben $0,3995 \mathrm{~g} \mathrm{CO}_{2}$ und $0,156 \mathrm{~g} \mathrm{H}_{2} \mathrm{O}$.

2. $0,135 \mathrm{~g}$ Substanz anderer Zubereitung gaben $0,3585 \mathrm{~g} \mathrm{CO}_{2}$ und $0,1415 \mathrm{~g} \mathrm{H}_{2} \mathrm{O}$.

Berechnet für $\mathrm{C}_{18} \mathrm{H}_{34} \mathrm{O}_{3}$ :

$$
\text { Gefunden: }
$$

C

$\mathrm{H}$

$$
\begin{aligned}
& 72,40 \\
& 11,51
\end{aligned}
$$

$\begin{array}{cc}1 . & 2 . \\ 72,15 & 72,42 \% \\ 11,58 & 11,73 \% .\end{array}$

Die zweite Substanz war frei von Schwefel.

Die Resultate der Analyse ergeben, dab die erhaltene Substanz nach ihrer Zusammensetzung einer Dioxystearinsäure weniger 1 Wassermolekül entspricht. Ihre Entstehung kann durch die Wirkung der Pottasche erklärt werden:

$$
\mathrm{C}_{18} \mathrm{H}_{34} \mathrm{O}_{2} \mathrm{H}_{2} \mathrm{SO}_{5}+\mathrm{K}_{2} \mathrm{CO}_{3}=\mathrm{C}_{18} \mathrm{H}_{34} \mathrm{O}_{3}+\mathrm{K}_{2} \mathrm{SO}_{4}+\mathrm{CO}_{2}+\mathrm{H}_{2} \mathrm{O} \text {. }
$$

Un ihre Natur zu bestimmen, wurde sie auf ihr Verhalten zu Wasser untersucht. $\mathrm{Zu}$ diesem Zwecke wurde sie mit Wasser im zugeschmolzenen Glasrohr 10 Stunden lang bis $120^{\circ}$ erhitzt. Nach Umkristallisieren des Produktes aus Alkohol erhielt man sebr wenig kristallinische Substanz, die nach dem Umkristallisieren aus Äther den Schmelzp. $128^{\circ}$ bis $129^{\circ}$, Erstarrungsp. $125^{\circ}-124^{\circ}$ zeigte. Beim Verseifen der Mutterlaugenreste mit $\mathrm{KOH}$ wurde wiederum Dioxystearin- 
372 Albitzky: Ǔber 0xydation ungesättigter Säuren etc.

säure erzielt, deren Schmelzpunkt nach dem Umkristallisieren aus Alkohol $127^{\circ}-129^{\circ}$, Erstarrungsp. $123^{\circ}-120^{\circ}$ war.

Die Bildung von Dioxystearinsäure durch Einwirkung von Wasser macht wahrscheinlich, daB die entstandene Substanz als eine Glycidsäure anzusehen ist.

Nach dem Ausziehen durch $\ddot{A}$ ther wurde die Lösung konzentriert und durch $\mathrm{H}_{2} \mathrm{SO}_{4}$ zerlegt, wobei sich ein Niederschlag bildete, der nach Umkristallisieren aus Alkohol zwei Fraktionen ergab: 1. vom Schmelzp. $128^{\circ}-131^{\circ}$, Erstarrungsp. $119^{\circ}-117^{\circ}$ und 2. Schmelzp. $119^{\circ}-122^{\circ}$, Erstarrungsp. $110^{\circ}$ bis $106^{\circ}$.

Das Filtrat wurde durch Äther extrahiert, der Rest begann nach Abdestillieren des Äthers im Exsikkator bald auszukristallisieren. Nach Behandlung mit wasserfreiem Äther blieb der größte Teil ungelöst (der Schmelztemperatur nach Dioxystearinsäure). Das nach Abdampfen des Äthers übrige Öl schied beim Aufbewahren im Exsikkator wiederum Kristalle aus, von denen es nochmals durch Lösen in wasserfreiem $\ddot{A}$ ther getrennt wurde. Zuletzt verblieb nur ein Öl, das nicht mehr Kristalle ausschied und sich in Äther leicht löste. $\mathrm{BaSO}_{4}$.

$0,178 \mathrm{~g}$ des Öls gaben $0,463 \mathrm{~g} \mathrm{CO}_{2}$ und $0,1825 \mathrm{~g} \mathrm{H}_{2} \mathrm{O}$.

$0,307 \mathrm{~g}$ des Öls gaben bei Bestimmung nach Carius $0,0505 \mathrm{~g}$

\begin{tabular}{cccc} 
& \multicolumn{2}{c}{ Berechnet für } & Gefunden: \\
& $\mathrm{C}_{18} \mathrm{H}_{34} \mathrm{O}_{2} \mathrm{H}_{2} \mathrm{SO}_{5}:$ & $\mathrm{C}_{18} \mathrm{H}_{98} \mathrm{O}:$ & \\
$\mathrm{C}$ & 54,48 & 68,27 & $70,93 \%$ \\
$\mathrm{H}$ & 9,17 & 11,49 & $11,48 \%$ \\
$\mathrm{~S}$ & 8,08 & - & $2,26 \%$
\end{tabular}

Die Resultate der Analyse erlauben nur den SchluB, daß die Substanz nicht einheitlich ist und, aller Wahrscheinlichkeit nach, ein Gemisch verschiedener Substanzen von anhydridischem Charakter vorstellt. Bei vielen Versuchen ergaben Substanzen, die aufgehört hatten Dioxystearinsäure auszuscheiden, ähnliche Resultate. Die Quantität des Kohlenstoffs schwankte von $68,65 \%-70,32 \%$, des Wasserstoffs von $10,93 \%-12,2 \%$, des Schwefels von $0,69 \%-2,29 \%$.

In bezug auf die Eigenschaften dieser Riuckstände kann nur gesagt werden, daß sie, frisch erhalten, sich in Äther 
Albitzky: Über Oxydation ungesättister Säuren өtc. 373

leicht lösen, die Fähigkeit besitzen, mit Wasser Emulsionen zu geben, aus denen beim Erhitzen im zugeschmolzenen Rohre oder beim Kochen mit $\mathrm{K}_{2} \mathrm{CO}_{3}$, wenn auch wenig, Dioxystearinsäure gewonnen wird. Beim längeren Aufbewahren erstarren sie zu einer gallertartigen Masse, die sich auch im kochenden Alkohol nicht löst und nur bei andauerndem Kochen in alkoholischem $\mathrm{KOH}$ in Lösung übergeht, aus der später etwas Dioxystearinsäure erhalten werden kann.

Es bleibt noch übrig, über einige Versuche zu berichten, die zur Ausscheidung des Zwischenproduktes führen sollten. Bei der Neutralisation durch Pottasche war beobachtet worden, dab nach einiger Zeit aus der Lösung ein dickflüssiges Öl niederfiel, das sich bei weiterem Zusatz von Pottasche wieder löste. Auf gewöhnliche Weise wurde die Lösung hergestellt, vorsichtig bis zum Ausfallen des Öles neutralisiert und das Öl durch Ather extrahiert. Aber auch diese Versuche mißlangen. Das extrahierte Öl begann beim Aufbewahren im Exsikkator von selbst Dioxystearinsäure auszuscheiden, und schlieBlich verblieben Rückstände, die annähernd dieselben Eigenschaften besaßen, wie schon oben beschrieben.

Bei Oxydation der höheren ungesättigten Säuren mit Kaliumpermanganat bilden sich außer Dioxysäuren noch zweibasische Säuren, die sich im Wasser lösen, z. B. die Azelaïnsäure. Da die Möglichkeit ihrer Bildung auch bei der Oxydation durch das Carosche Reagens vorauszusetzen war, so bemühte man sich, sie unter den Produkten der Reaktion aufzufinden, aber ihr Nachweis gelang nicht. AuBerdem zeigte auch das Gewicht des Produktes vor dem Umkristallisieren aus Alkohol an, daß es in Wasser unlöslich ist.

Was die Erklärung der Reaktion anbetriff, so muB man voraussetzen, daß bei Einwirkung des Caroschen Reagens auf eine ungesättigte Säure eine Verbindung entsteht, analog der, die bei der Einwirkung uer Schwefelsäure auf Ölsäure sich bildet. Diese Verbindung ist nicht haltbar, denn sie zerfällt schon durch eine minimale Wassermenge teils in Dioxysäure und Schwefelsäure, teils erfährt sie eine tiefer greifende Veränderung, wobei anhydridartige Substanzen sich bilden. Für 
374 Albitzky: Ũber Oxydation ungesä,ttigter Säuren etc. eine solche Erklärung spricht auch die' Löslichkeit des erhaltenen Produktes in Wasser und das Fehlen der Spaltungsprodukte, z. B. der zweibasischen Säuren. Man konnte natürlich erwarten, daß in den Rückständen, nachdem die Bildung der Dioxystearinsäure aufgehört hatte, eine genügende Quantität Schwefel enthalten sei, es wurden jedoch nicht mehr als 2,29\% gefunden. Dieses erklärt sich dadurch, daß diese Rückstände das Produkt einer tief greifenden :Veränderung sind; auch jene Schwefelsäure, die sich bei der Hydrolyse entwickelte, konnte bei der Behandlung mit Äther, beim Filtrieren u. s. w. leicht verloren gehen.

Die Reaktion der Oxydation kann auf folgende Weise ausgedrückt werden.

Wie die Ölsäure sich mit Schwefelsäure zu Stearinschwefelsäure vereinigt,

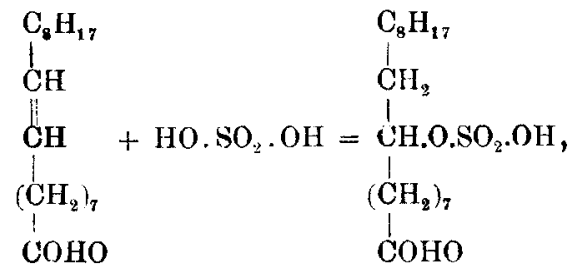

so bildet sich auch mit der Caroschen Säure infolge ihrer Addition der Einfach-Schwefelsäureester der Dioxystearinsäure :

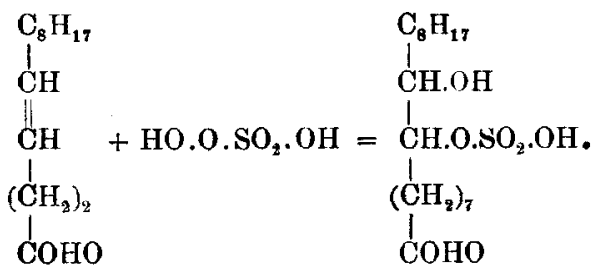

Ähnlich, wie die Stearinschwefelsäure durch Wasser in Oxystearinsäure und Schwefelsäure zerfällt:<smiles>O=CC(CCBr)C(CCBr)C(=O)OO</smiles> 
Albitzky: Über Oxydation ungesättigter Säuren etc. 375 so gibt auch der Ester der Dioxystearinsäure Dioxystearinsäure und Schwefelsäure:

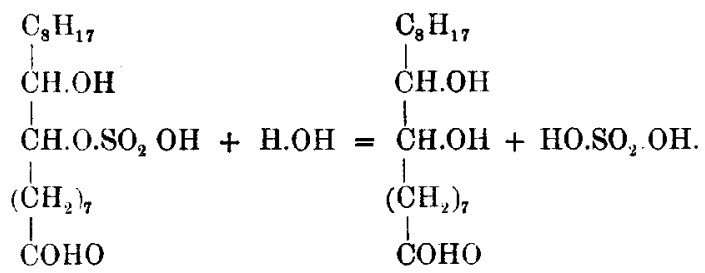

Eine ähnliche Erklärung gab $M$. Lw off ${ }^{1}$ ) für die Reaktion der Oxydation der Äthylenkohlenwasserstoffe mit schwacher Lösung von Kaliumpermanganat. Der Sinn seiner Erklärung wird an der Hand folgender Formeln verständlich, durch die er alle Umwandlungen bei der Oxydation ausdrückt:

1. $\left(\mathrm{MnO}_{2}\right) \mathrm{OOK}+\mathrm{mH}_{2} \mathrm{O}=\left(\mathrm{MnO}_{2}\right) \mathrm{OOH}+\mathrm{KOH}+(\mathrm{m}-1) \mathrm{H}_{2} \mathrm{O}$.

2.

$$
\mathrm{C}_{\mathrm{n}} \mathrm{H}_{2 \mathrm{n}}+\left(\mathrm{MnO}_{2}\right) \mathrm{OOH}=\mathrm{C}_{\mathrm{n}} \mathrm{H}_{2 \mathrm{n}} \succ_{\mathrm{OH}}^{\mathrm{O}\left(\mathrm{MnO}_{2}\right)}
$$

3.

$$
\begin{aligned}
& \mathrm{Cn}_{2} \mathrm{H}_{2}\left\langle\begin{array}{l}
\mathrm{O}\left(\mathrm{MnO}_{2}\right) \\
\mathrm{OH}
\end{array}+(\mathrm{m}-1) \mathrm{H}_{2} \mathrm{O}=\mathrm{C}_{\mathrm{n}} \mathrm{H}_{2 n} \succ_{\mathrm{OH}}^{\mathrm{OH}}+\left(\mathrm{MnO}_{2}\right) \mathrm{OH}\right. \\
& +(m-2) \mathrm{H}_{2} \mathrm{O} \text {. }
\end{aligned}
$$

Was den Zeitmoment der Umlagerung anbetrifft, durch die sich die Dioxysäure, die der bei Oxydation mit Kaliumpermanganat erhaltenen stereoisomer ist, bildet, so mub angenommen werden, daß es derselbe ist, in dem die Addition der Caroschen Säure vor sich geht. Bei einer Erklärung der Reaktion der Entstehung anormaler Dioxystearinsäure aus Chloroxystearinsäure, infolge der Einwirkung von $\mathrm{KOH}$ unter Erwärmen, kann noch die Voraussetzung zugelassen werden, dab die Umlagerung entweder im Moment der Addition von $\mathrm{HClO}$ oder der Substitution von $\mathrm{Cl}$ durch $\mathrm{OH}$ erfolgt; im gegebenen Falle aber ist die Annahme schwierig, daß Wasser ohne Erwärmen eine ähnliche Umlagerung veranlassen könnte.

Diese Umlagerungen können für Elaidinsäure durch folgende Konfigurationen ausgedrückt werden:

1) Journ. d. russ. ph. Chem. Ges. 21, 351. 
376 Albitzky: Über 0xjdation ungesättigter Säuren etc.

$$
\begin{gathered}
\mathrm{H}-\mathrm{C}-\left(\mathrm{CH}_{2}\right)_{7}-\mathrm{CH}_{3} \\
\mathrm{H}-\mathrm{C}-\left(\mathrm{CH}_{2}\right)_{7}-\mathrm{COHO}
\end{gathered}+\mathrm{HO}-\mathrm{SO}_{2} . \mathrm{SO}_{2} \mathrm{OH}=\begin{gathered}
\mathrm{H}-\mathrm{C}-\left(\mathrm{CH}_{2}\right)_{2}-\mathrm{CH}_{3} \\
\mathrm{H}-\mathrm{C}-\left(\mathrm{CH}_{2}\right)_{7}-\mathrm{COHO}, \\
! \\
\mathrm{OH}
\end{gathered}
$$

der entstandene Ester lagert sich um in

$$
\begin{gathered}
\mathrm{O}-\mathrm{SO}_{2} \mathrm{OH} \\
\mathrm{CH}_{3}-\left(\mathrm{CH}_{2}\right)_{7}-\mathrm{C}-\mathrm{H} \\
\mathrm{H}-\mathrm{C}-\left(\mathrm{CH}_{2}\right)_{7}-\mathrm{COHO} \\
\mathrm{OH}^{\mathrm{O}}
\end{gathered}
$$

Dieser letztere geht durch Drehung in die beständige Form über und gibt durch Substitution des Restes $\mathrm{O}_{\mathrm{SO}} \cdot \mathrm{OH}$ mittels $\mathrm{OH}$ :

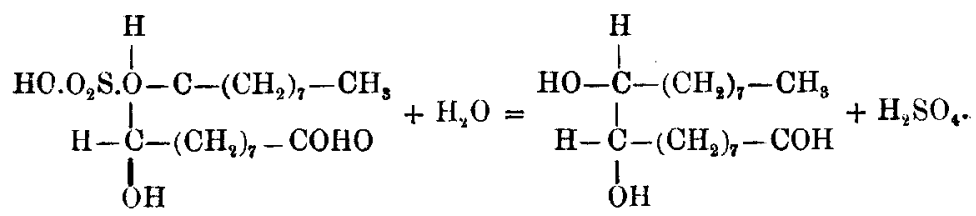

